\title{
2D SDS PAGE in Combination with Western Blotting and Mass Spectrometry Is a Robust Method for Protein Analysis with Many Applications
}

\author{
Nancy Kendrick, Costel C. Darie, Matt Hoelter, \\ Ginny Powers, and Jon Johansen
}

\section{Abstract}

Two-dimensional sodium dodecyl sulfate polyacrylamide gel electrophoresis (2D SDS PAGE) is a method that separates proteins according to their isoelectric points in the first dimension and molecular masses in the second dimension. Evidence is provided that 2D SDS PAGE is reproducible, robust and compatible with SDS in both dimensions including isoelectric focusing in tube gels, the first dimension. The 2D gel pattern of rat liver microsomes shows more detail and sharper spot outlines when dissolved in SDS buffer with heating than in urea buffer and is better yet when dissolved in a mixture of both buffers. Quantification of 60 proteins in rat liver cytosol over a wide range of $\mathrm{pI}$ and MW gave linear plots of spot density versus total protein for loads of 200,400 and $600 \mu \mathrm{g}$ protein dissolved in SDS buffer and run in triplicate on 2D gels (Average $\mathrm{R}^{2}=0.987$ ). Examples of biomedical applications are provided in which $2 \mathrm{D}$ proteins of interest found by comparing stained or western blotted 2D gel patterns were identified by mass spectrometry (MS).

\section{Keywords}

Protein analysis - Electrophoresis - 2D electrophoresis · Western blotting

The original version of this chapter was revised. The correction to this chapter is available at https://doi.org/10.1007/978-3-030-15950-4_47

N. Kendrick $(\bowtie) \cdot$ M. Hoelter $\cdot$ G. Powers $\cdot$ J. Johansen

Kendrick Laboratories, Inc., Madison, WI, USA

e-mail: nancy@kendricklabs.com; http://www.kendricklabs.com

C. C. Darie

Biochemistry \& Proteomics Group, Department of Chemistry \& Biomolecular Science, Clarkson University, Potsdam, NY, USA

\begin{tabular}{ll}
\hline Abbreviations & \\
1D & $\begin{array}{l}\text { One-dimensional } \\
\text { Two-dimensional sodium dodecyl sulfate } \\
\text { 2D SDS PAGE }\end{array}$ \\
2D & Two-dimensional \\
$\alpha$-syn & Alpha-synuclein \\
IEF & Isoelectric focusing \\
MS & Mass spectrometry \\
nanoLC-MS/MS & Nanoliquid chromatography tandem MS \\
NP-40 & Nonidet-P-40 aka IGEPAL \\
OSA & Obstructive sleep apnea \\
pI & Isoelectric point \\
PTM & Post-translational modification \\
STK16 & Serine/threonine kinase 16
\end{tabular}

\subsection{Introduction}

Two-dimensional electrophoresis (2D PAGE), in place since 1975 [1], is a biochemical method for separating complex mixtures of proteins into individual species. Proteins are separated by charge in the first dimension using isoelectric focusing (IEF) then by size using sodium dodecyl sulfate polyacrylamide gel electrophoresis (SDS PAGE). The resulting starburst pattern of protein spots may be visualized on final 2D gels by autoradiography, protein stains such as silver, Coomassie and Sypro Ruby, or by western blotting (WB). The gel patterns may be compared by eye or digitized images compared using commercial 2D software such as Progenesis SameSpots from TotalLab. Proteins of interest may be cut from stained gels and identified by MS. There are many applications for this approach.

Two major variations of $2 \mathrm{DE}$ are currently in use that differ in IEF protocol. There are advantages and disadvantages 
Fig. 33.1 Carbamylated CPK standard dissolved in either SDS or $9 \mathrm{M}$ urea buffer before IEF. The arrow marks the lower isoform of tropomyosin, a 2D marker of MW $33 \mathrm{kDa}$ and pI 5.2. Preparation in SDS buffer does not affect separation of the single charge Carb-CPK isoforms

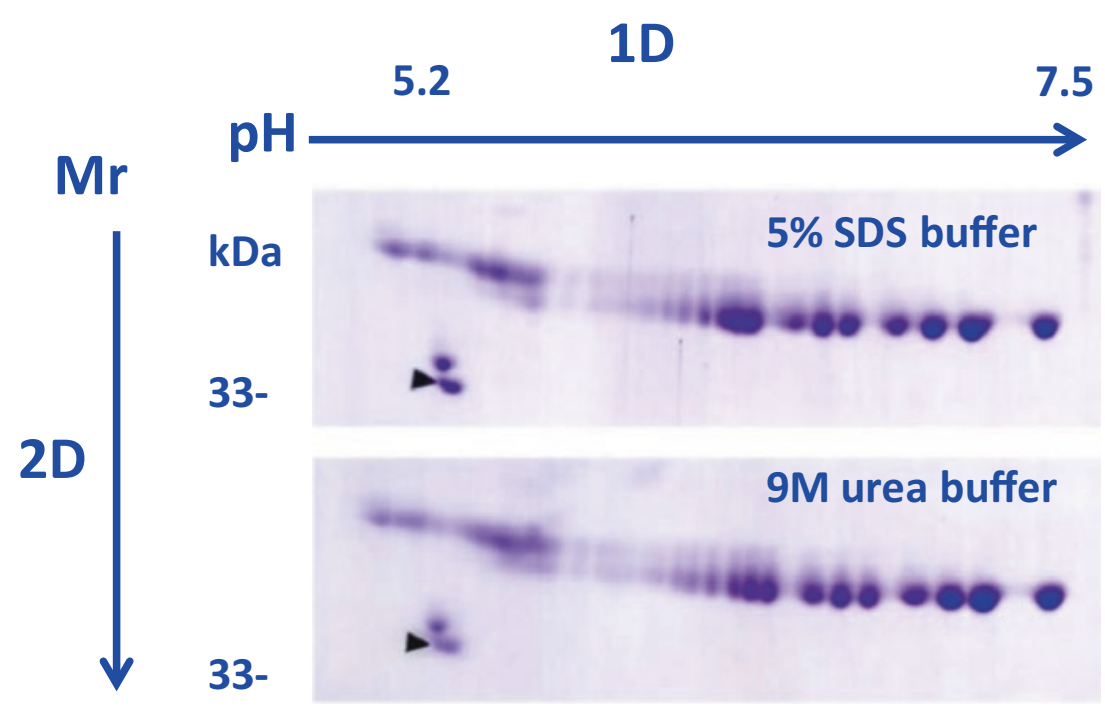

to each. In the newest and most common variant, 2D PAGE, the $\mathrm{pH}$ gradient is fixed in place on commercially available solid supports called immobilized $\mathrm{pH}$ gradient (IPG) strips [2]. IPG strips are technically convenient to run but are incompatible with the detergent SDS, by far the best reagent for dissolving membrane proteins $[3,4]$. Sample preparation for IPG strips utilizes nonionic detergents and urea that work fine for soluble proteins but less so for membrane proteins that comprise about $30 \%$ of the human genome [5]. For membranous samples, whole cell lysates for example, a centrifugation step is often included to remove insoluble "cell debris", causing unknown losses. Protein losses occur at different stages of the IPG strip procedure as well $[6,7]$.

The 2D classic method, first described by O'Farrell in 1975, uses acrylamide tube gels polymerized with commercial carrier ampholines for IEF. A pH gradient forms when voltage is applied; ampholines and proteins migrate to a steady-state position during the overnight IEF step. The tube gel is extruded, incubated with SDS buffer, and fixed on top of a slab gel prior to SDS PAGE. The classic method is technically difficult but has the huge advantage of being compatible with SDS as clearly demonstrated by the Andersons [8]. Tissue samples are homogenized in SDS buffer with heating until the solution clarifies; centrifugation is usually unnecessary.

Our laboratory (Kendrick Labs) validated 2D SDS PAGE, where the classic method is used for samples prepared in SDS, with regard to reproducibility and linearity of response in 1989 [9]. Since then, all aspects of 1D and 2D SDS PAGE have been standardized with written standard operating procedures (SOPs) subject to change control. Electrophoresis testing is performed by trained Biochemists using validated equipment and software. The final critical step, identifying proteins of interest using MS analysis, is carried out at an outside core facility.
Evidence is presented here that SDS is compatible with IEF in tube gels, and that using SDS for sample preparation imparts robustness to the classic 2D PAGE method. In combination with WB and MS, 2D SDS PAGE is a useful orthogonal tool for sorting out many biological processes, especially those involving post-translational modifications (PTMs) of proteins.

\subsubsection{Evidence of IEF Compatibility with SDS}

2D SDS PAGE (carrier ampholine 2D) is compatible with SDS, the best reagent by far for solubilizing proteins [4]. The method, originally developed by O'Farrell [1], was shown to have SDS compatibility by the Andersons [8] and refined at Kendrick Labs [9].

As demonstrated by the Andersons in 1988, SDS is stripped off proteins by the nonionic detergent NP-40 included in the tube gel polymerization mixture. During overnight IEF, micelles containing NP-40 and SDS migrate to the extreme acid end of the tube gel where they form a visible bulb that is cut off and discarded [8].

\subsubsection{SDS Versus Urea Buffer for a Purified pI Standard}

While it is difficult to demonstrate that SDS is stripped off every protein, 2D SDS PAGE (abbreviated from here on as 2D) patterns are quite reproducible. Figure 33.1 shows the 2D pattern of carbamylated creatine phosphokinase (CarbCPK), an IEF pI standard prepared by urea carbamylation to generate a collection of isoforms differing by one charge [10]. Isoelectric points of the charge isoforms range from $\mathrm{pH} 5.0$ on the left to 7.5 on the right. The 2D pattern of CarbCPK standard dissolved in urea is virtually identical to that dissolved in SDS buffer. 


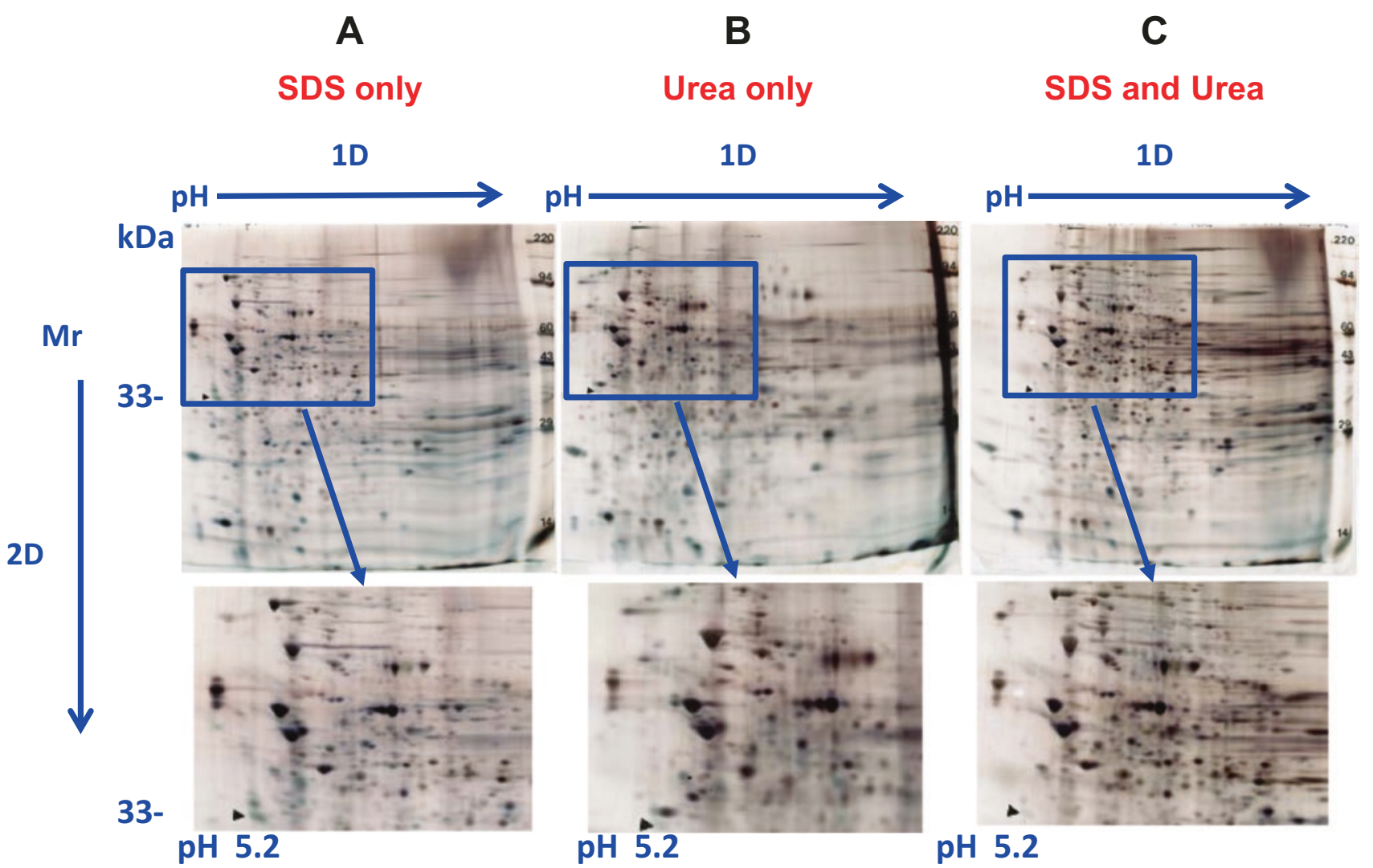

Fig. 33.2 Rat liver microsomes prepared with and without SDS. For this figure, microsomes (pellet obtained after homogenization, low speed spin to remove cell debris, and then $100,000 \times \mathrm{g}$ spin) were purchased and dissolved in (a). SDS buffer with heating, (b) urea buffer

\subsubsection{SDS Versus Urea Buffer for a Membrane Fraction}

The carb-CPK protein standard shown in Fig. 33.1 is water soluble and dissolves easily in either SDS or urea buffer. Roughly $30 \%$ of protein-coding genes in the human genome code for membrane proteins that have hydrophobic domains and are often difficult to dissolve without SDS [5]. Figure 33.2 shows 2D patterns of a rat liver microsome sample, derived from the endoplasmic reticulum of hepatic cells prepared with and without SDS buffer. The same sample was: (A) dissolved in SDS buffer sample and the tube heated in a boiling water bath for $5 \mathrm{~min}$, (B) dissolved in $9 \mathrm{M}$ urea plus 2\% NP-40 without heating, and (C) dissolved in both, i.e. an aliquot of the sample from $A$ was diluted 1:1 with urea buffer from B. The final concentration was $2.5 \%$ SDS, $4.5 \mathrm{M}$ urea and $1 \% \mathrm{NP}-40$.

The expanded sections show that the 2D pattern from microsomes dissolved in SDS buffer show more detail and sharper spots that those dissolved in urea buffer. The best pattern, however, was obtained by combining reagents i.e. adding urea buffer after prior preparation without heating, or (c) SDS buffer with heating followed by 1:1 dilution with urea buffer. The $13 \times 15 \mathrm{~cm}$ silver-stained $2 \mathrm{D}$ gels were loaded with $50 \mu \mathrm{g}$ protein. Patterns from microsomes prepared with both SDS and urea, show the best resolution

with SDS buffer and heating. Note that heating proteins in the presence of urea causes carbamylation artifacts and should be avoided.

\subsubsection{Protein Quantification Using 2D SDS PAGE}

Using SDS for 2D sample preparation improves method robustness as shown in the following experiment. A complex mammalian sample, rat liver cytosol, was diluted with buffer containing $2.5 \% \mathrm{SDS}+4.5 \mathrm{M}$ urea before running on large format 2D gels $(20 \times 20 \mathrm{~cm})$ in triplicate at loads of 200, 400 and $600 \mu \mathrm{g}$. The gels were Coomassie blue stained and scanned with a laser densitometer verified to be linear over 0-3 OD. Sixty polypeptide spots, chosen over a wide range of pI and MW, were quantified on each gel with Progenesis SameSpots software from TotalLab.

Results were tabulated in two ways: (1) as integrated spot density above background within the outline and (2) as spot percentage of total density of all spots combined. Figure 33.3 shows the 2D gel pattern for a $600 \mu \mathrm{g}$ load along with spot numbering. 


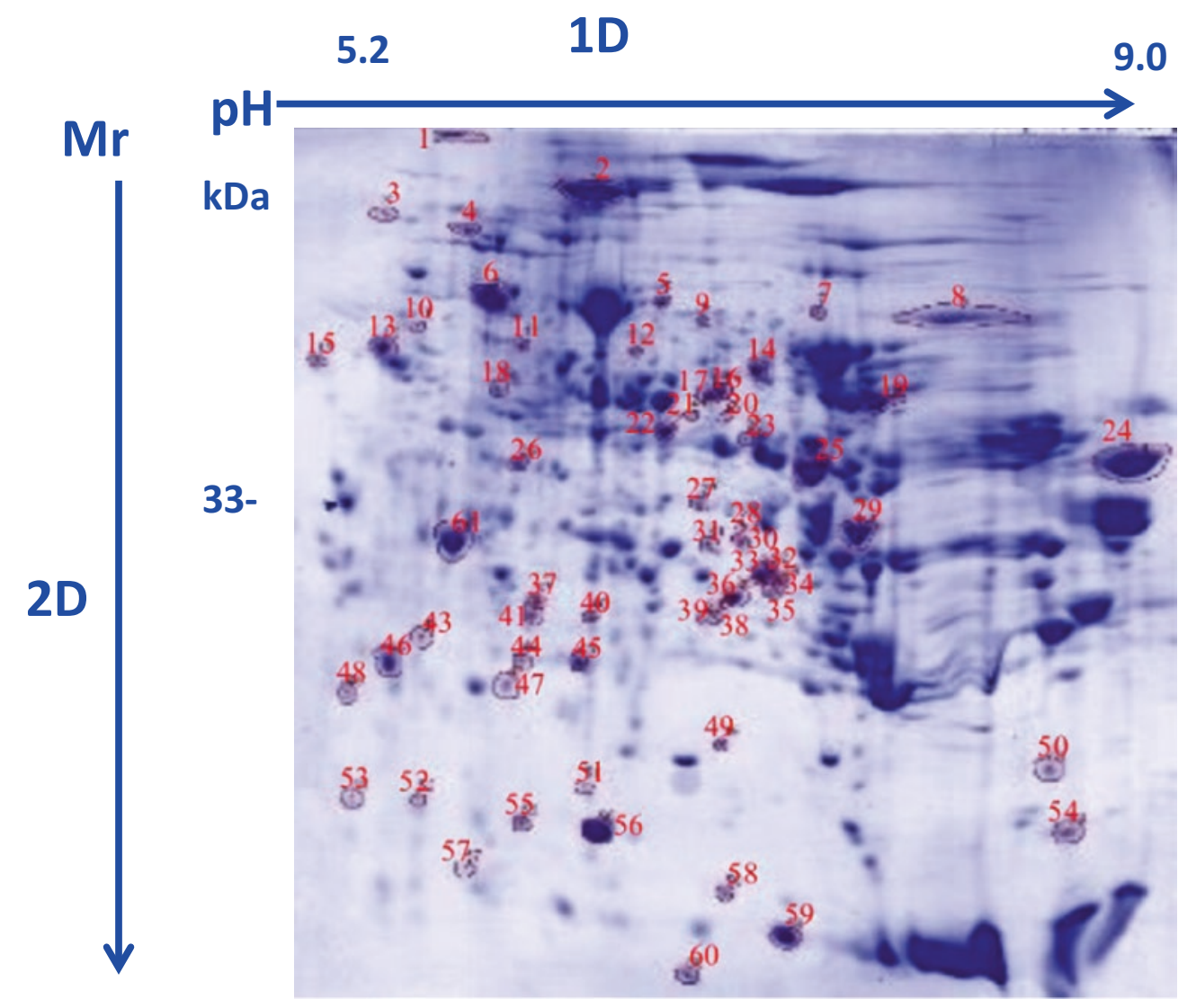

Fig. 33.3 Rat liver cytosol $(600 \mu \mathrm{g})$ prepared with SDS buffer plus urea showing outlines of 60 quantified protein spots

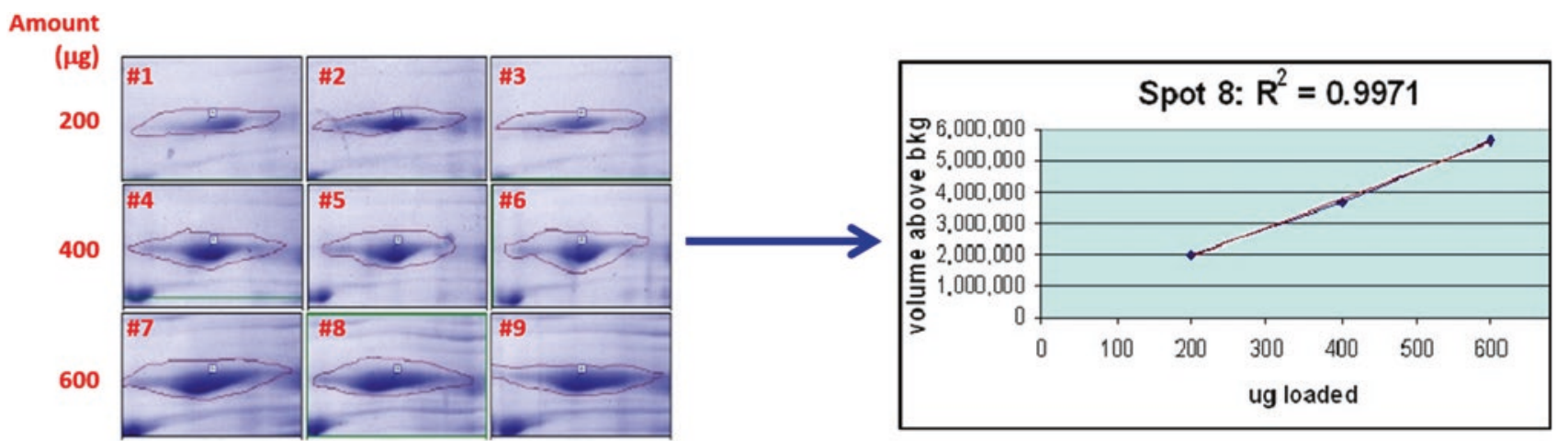

Fig. 33.4 Quantification of protein 8 based on spot volume. Left: Montage of Spot 8 outlines taken from the nine 2D gels to show the 200 (top), 400 (middle) and $600 \mu \mathrm{g}$ (bottom) patterns. Right: Graph showing a plot of average integrated density from triplicate gels versus load for spot 8

Figure 33.4 shows outlines for a single spot along with the corresponding plot of average spot volume ( $\mathrm{n}=3$ gels) versus total protein load. Similar plots were determined for each of the 60 proteins.

Absolute spot volume is useful for determining linearity of the method within a run but is seldom used for comparing samples prepared and run on different days. For the latter, measurements are normalized to correct for gel-to-gel load- ing and staining differences by expression as spot percentages, defined as spot volume taken as a percentage of total volume of all spots combined. Spot percentages are largely independent of sample load.

Table 33.1 shows results taken from both ways of expressing data. $\mathrm{R}^{2}$ values for each plot are provided as a measurement of linearity of spot volume versus load. The Coefficient of Variation $(\mathrm{CV}=\mathrm{SD} /$ mean $\times 100)$ is provided 
Table 33.1 Spot Volume and Spot \% results obtained from 60 polypeptide spots on 9 gels

\begin{tabular}{|c|c|c|c|c|c|c|c|c|c|c|c|c|c|c|}
\hline Spot & $\mathrm{pI}$ & $\begin{array}{l}\text { MW } \\
(\mathrm{kDa})\end{array}$ & $\begin{array}{l}\mathrm{R}^{2} \text { value } \\
\text { for plot }\end{array}$ & $\begin{array}{c}\mathrm{CV}(\mathrm{n}=9) \\
\text { Spot } \%\end{array}$ & Spot & $\mathrm{pI}$ & $\begin{array}{l}\text { MW } \\
(\mathrm{kDa})\end{array}$ & $\begin{array}{l}\mathrm{R}^{2} \text { value } \\
\text { for plot }\end{array}$ & $\begin{array}{c}\text { CV }(n=9) \\
\text { Spot } \%\end{array}$ & Spot & $\mathrm{pI}$ & $\begin{array}{l}\text { MW } \\
(\mathrm{kDa})\end{array}$ & $\begin{array}{l}\mathrm{R}^{2} \text { value } \\
\text { for plot }\end{array}$ & $\begin{array}{c}\text { CV }(n=9) \\
\text { Spot } \%\end{array}$ \\
\hline 1 & $\sim 5.7$ & 300 & 0.990 & $12 \%$ & 21 & 6.8 & 47.7 & 0.999 & $17 \%$ & 41 & 6.0 & 28.7 & 0.993 & $14 \%$ \\
\hline 2 & 6.3 & 177 & 0.999 & $16 \%$ & 22 & 6.7 & 44.4 & 0.991 & $15 \%$ & 61 & 5.7 & 33.8 & 0.997 & $6 \%$ \\
\hline 3 & 5.4 & 131 & 0.974 & $13 \%$ & 23 & 7.1 & 43.6 & 0.971 & $24 \%$ & 43 & 5.6 & 27.0 & 0.999 & $10 \%$ \\
\hline 4 & 5.7 & 118 & 0.786 & $20 \%$ & 24 & 9.4 & 41.0 & 0.989 & $8 \%$ & 44 & 6.0 & 26.2 & 0.995 & $26 \%$ \\
\hline 5 & 6.7 & 79.9 & 0.998 & $12 \%$ & 25 & 7.4 & 40.9 & 1.000 & $4 \%$ & 45 & 6.2 & 26.2 & 0.991 & $17 \%$ \\
\hline 6 & 5.8 & 78.4 & 0.989 & $9 \%$ & 26 & 6.0 & 40.5 & 0.994 & $8 \%$ & 46 & 5.4 & 25.7 & 0.997 & $5 \%$ \\
\hline 7 & 7.4 & 78.3 & 0.998 & $14 \%$ & 27 & 6.8 & 37.6 & 1.000 & $13 \%$ & 47 & 5.9 & 24.8 & 0.999 & $9 \%$ \\
\hline 8 & 8.1 & 76.1 & 0.997 & $4 \%$ & 28 & 7.1 & 32.3 & 0.984 & $23 \%$ & 48 & 5.3 & 24.4 & 0.998 & $12 \%$ \\
\hline 9 & 6.9 & 73.9 & 0.984 & $16 \%$ & 29 & 7.5 & 35.9 & 0.985 & $12 \%$ & 49 & 6.9 & 22.0 & 0.996 & $16 \%$ \\
\hline 10 & 5.5 & 67.9 & 0.982 & $8 \%$ & 30 & 7.1 & 35.0 & 0.992 & $8 \%$ & 50 & 8.9 & 21.2 & 0.992 & $24 \%$ \\
\hline 11 & 6.0 & 65.4 & 0.994 & $8 \%$ & 31 & 6.9 & 34.3 & 0.998 & $18 \%$ & 51 & 6.3 & 19.5 & 0.999 & $28 \%$ \\
\hline 12 & 6.5 & 64.0 & 0.998 & $15 \%$ & 32 & 7.2 & 32.8 & 0.996 & $15 \%$ & 52 & 5.6 & 18.6 & 0.999 & $22 \%$ \\
\hline 13 & 5.4 & 60.8 & 0.990 & $4 \%$ & 33 & 7.1 & 32.6 & 0.977 & $11 \%$ & 53 & 5.3 & 18.3 & 0.994 & $15 \%$ \\
\hline 14 & 7.1 & 60.3 & 0.985 & $10 \%$ & 34 & 7.2 & 31.9 & 1.000 & $9 \%$ & 54 & 9.0 & 17.9 & 0.967 & $60 \%$ \\
\hline 15 & 4.9 & 58.4 & 1.000 & $14 \%$ & 35 & 7.2 & 31.1 & 0.994 & $37 \%$ & 55 & 6.0 & 17.5 & 1.000 & $12 \%$ \\
\hline 16 & 7.0 & 54.1 & 0.984 & $16 \%$ & 36 & 7.0 & 30.5 & 0.998 & $7 \%$ & 56 & 6.3 & 17.0 & 1.000 & $7 \%$ \\
\hline 17 & 6.9 & 52.8 & 0.978 & $24 \%$ & 37 & 6.1 & 29.5 & 0.972 & $16 \%$ & 57 & 5.7 & 14.8 & 1.000 & $36 \%$ \\
\hline 18 & 5.9 & 52.6 & 0.969 & $11 \%$ & 38 & 6.9 & 29.4 & 0.981 & $10 \%$ & 58 & 6.9 & 14.5 & 0.998 & $6 \%$ \\
\hline 19 & 7.6 & 52.0 & 0.979 & $8 \%$ & 39 & 6.9 & 28.8 & 1.000 & $22 \%$ & 59 & 7.2 & 10.1 & 0.997 & $13 \%$ \\
\hline 20 & 7.0 & 48.2 & 0.958 & $34 \%$ & 40 & 6.3 & 28.7 & 0.999 & $5 \%$ & 60 & 6.7 & 4.1 & 0.985 & $13 \%$ \\
\hline & & & & & & & & & \multicolumn{4}{|c|}{ Average All Spots $(n=60)$} & 0.987 & $15 \%$ \\
\hline
\end{tabular}

Spot outlines are shown in Fig. 33.4. Spots with high error $\left(\mathrm{CV}\right.$ for spot percentage $>20$ or $\mathrm{R}^{2}$ values from the plots $\left.<0.95\right)$ are highlighted with colors. Pink indicates a protein aggregation problem. Orange indicates a spot splitting problem. Green indicates that the spot was faint on the $200 \mu \mathrm{g}$ load gels. The fainter the spot, the higher the error

as a measure of variability of spot \% values between the nine gels.

The average $\mathrm{R}^{2}$ value for the 60 spots was 0.987 indicating good correspondence between individual proteins and total sample load. When the latter is doubled, the individual spot volumes double as well for Coomassie blue, a quantitative stain. A few spots were outliers for varying reasons. Spot 4 showed irreproducibility that we speculate was due to protein aggregation. It gave about the same spot volume at the 400 and $600 \mu$ g loads. Spots 17, 23 and 35 showed spot splitting problems. Isoforms of the same or adjacent proteins are merging at higher loads; small shifts in the splitting line increase error. Five remaining spots were faint on the $200 \mu \mathrm{g}$ gels; fainter spots have higher error. Overall, we have great correspondence between individual proteins and total sample load.

\subsubsection{D SDS PAGE Followed by MS Is Useful for Identification of Specific Changing Proteins in Complex Mixtures}

The human genome is an invariant blueprint present in all cells and tissues of each person. In contrast, there is no constant human "proteome"; rather, the protein composition of cells and tissues varies with function and developmental stage. Deciphering how genomic transcription translates into proteins that vary dramatically between tissues and are altered in disease states is quite difficult. Using a concerted approach of several orthogonal techniques rather than one alone holds promise for giving the best outcome [11].

\subsubsection{Proteins Changes Between Samples May Be Found by Comparing 2D Stained Patterns for Differences and Identified by MS as Shown in Two Examples Below}

Sleep apnea: Lux et al. studied protein dysregulation in obstructive sleep apnea (OSA) by comparing 2D patterns from rat atrial tissue induced to have OSA via a surgical implant versus normal control tissue. Over 2000 protein spots were compared for differences of which 208 differing spots were identified by nanoLC-MS/MS. Figure 33.5 shows a silver-stained 2D gel atrial pattern from a severe apnea sample. Spots increased in averaged severe apnea vs averaged control are outlined in blue; decreased spots are outlined in red.

In total, the data shows that enzymes involved in glycolysis, beta-oxidation, electron transport chain and Krebs cycle 

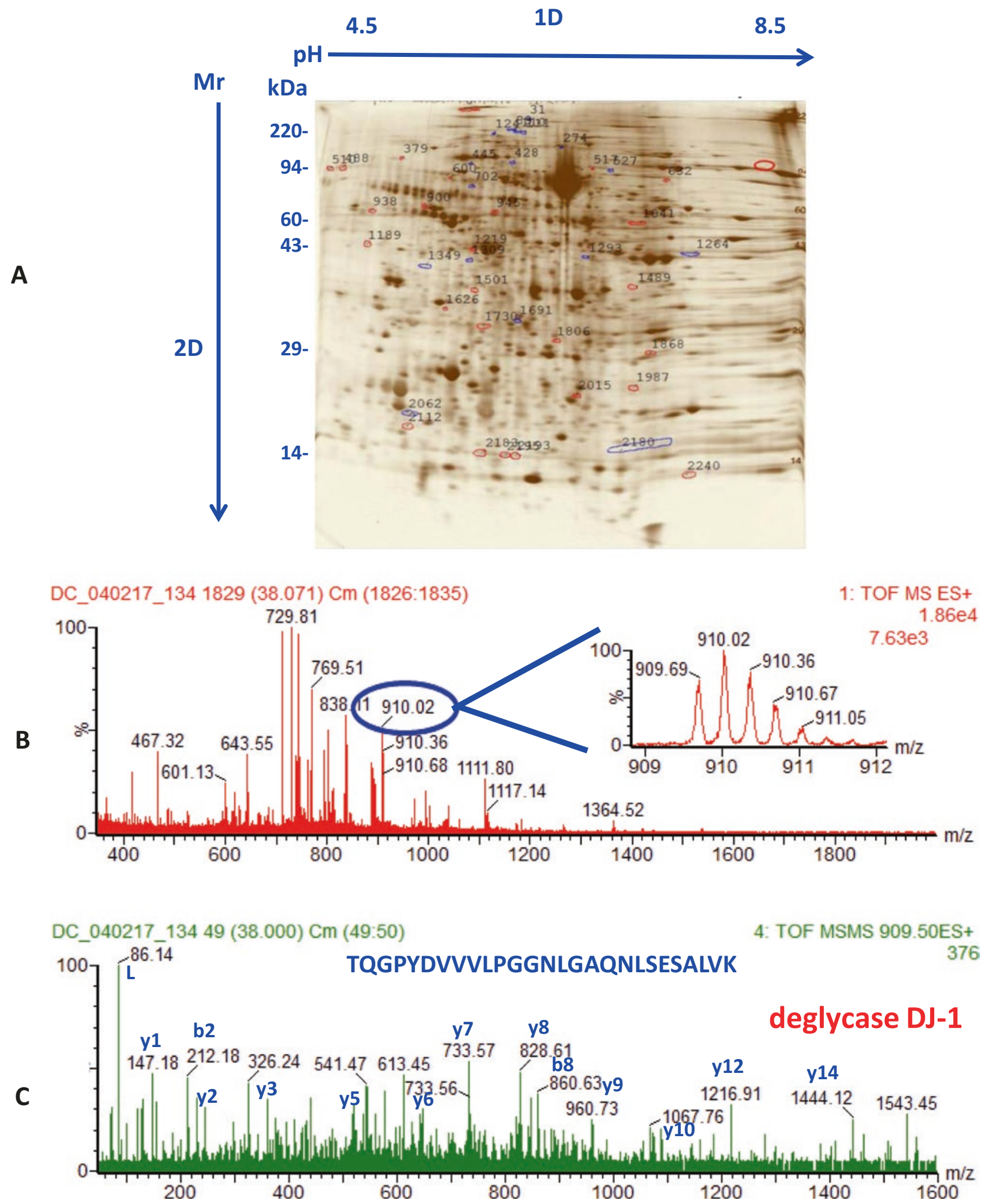

Fig. 33.5 (a) Image of 2D SDS PAGE gel run with atrial tissue from a severe OSA animal showing difference versus control. Spots increased in averaged severe OSA vs averaged control are outlined in blue; decreased spots are outlined in red. (b) The MS spectrum that contains the precursor ion with $\mathrm{m} / \mathrm{z} 909.69(3+)$ corresponds to peptide TQGPYDVVVLPGGNLGAQNLSESALVK, which is part of protein deglycase DJ-1 isoform 2. (c) The MS/MS fragmentation of precursor ion with $\mathrm{m} / \mathrm{z} 909.69(3+)$ produced a series of $\mathrm{b}$ and $\mathrm{y}$ ions whose analysis confirmed the sequence of peptide TQGPYDVVVLPGGN LGAQNLSESALVK and therefore the identity of protein deglycase DJ-1 isoform 2 
were downregulated in apnea. This model adds to understand of OSA and may serve to elucidate cardiac effects of OSA as well [12].

MPS 1: Ou et al. used 2D SDS PAGE to investigate mucopolysaccharidosis type 1 (MPS 1), a disease where alpha-L-iduronidase is deficient, causing an accumulation of undegraded glycosaminoglycans (GAG). To identify potential biomarkers and further characterize neuropathology, brains from MPS 1 and control mice were analyzed by 2D SDS PAGE. A total of 2055 protein spots were compared. Of these, 25 spots corresponding to 50 different proteins with a fold change of $>3.5$-fold and a $\mathrm{p}$ value $<0.05$ were identified by nanoLC-MS/MS. The MS spectrum of protein 1351, Sirtuin SIR2L2 is shown as an example in Fig. 33.6 along with a $2 \mathrm{D}$ silver-stained gel image.

The identified proteins were involved in metabolism, neurotransmission and cytoskeleton. STXBP1, a regulator of synaptic vesicle fusion and docking, was downregulated suggesting impaired synaptic transmission. Notably, alterations in proteins associated with cell death, ubiquitin or inflammation were not detected [13].

\subsubsection{Proteins with PTMs Involved in Disease Processes May Be Found by Comparing 2D Western Blots and Identified by MS}

Mammalian proteins have many PTMs that are important in cell development and diseases [14]. Trying to identify PTMs on proteins in whole cell lysates using MS alone can be difficult. Large numbers and amounts of interfering proteins cause problems. Using 2D SDS PAGE WB with high affinity antibodies is another way of looking at PTM subsets. Once found by WB, proteins may be subsequently identified by MS. Serine/ threonine phosphorylation is used as an example.

\section{Serine/Threonine Phosphorylation}

Olsen et al., using MS to study synchronized cultured cells, concluded that as much as $70 \%$ of cell proteins $(5192 / 6695$ proteins analyzed) are phosphorylated during the cell division cycle [15]. The human genome contains over 500 kinases [16]. Some are well understood, but the role of many kinases in cellular differentiation and disease processes remains to be worked out. Direct identification of phosphoserine (pSer) and phosphothreonine (pThr) by MS is certainly possible as shown in Fig. 33.7.

In the case of whole cell lysates, direct detection of pSer and pThr residues becomes difficult because of the vast number of interfering proteins. In the following example, LopezCorel et al. directly detected these PTMs by pSer/pThr WB with follow up identification of the proteins by MS. This group's focus was on the interesting problem of how the plasma membranes of hepatocytes become polarized.
Hepatocyte apical membranes, which face the bile have a different contingent of proteins than the basolateral membranes which face the blood. This group had been using WIF-B cells, a well-characterized hepatic cell model, to study the role of MAL2 (myelin and lymphocyte protein 2) in regulating hepatic polarized protein sorting. They learned that serine/threonine kinase 16 (STK16) was a binding partner of MAL2.

In order to find STK16 substrates, Lopez-Coral et al. compared post-nuclear supernatants of WT WIF-B cells versus a kinase-dead strain (E202A) for differences in pSer/ pThr-proteins using 2D WB as shown in Fig. 33.8. Red outlines indicate pSer/pThr-proteins decreased in E202A, i.e. putative STK16 substrates. A total of 28 proteins identified from the 16 spots included WDR1, spot 101, that contained two STK16 consensus sequences. The MS sequence of spot 101 is shown in Fig. 33.8. They concluded that WDR1 is a phosphoprotein that regulates secretion [17].

\section{Additional Examples}

Aslebagh et al. used 2D SDS PAGE in combination with MS to determine if milk proteins were affected by breast cancer [18]. Silver-stained 2D SDS PAGE patterns of milk from a woman diagnosed with breast cancer were compared to those of normal milk from the other breast for differences. Statistically different spots were picked from matched Coomassie blue-stained gels for protein digestion followed by nanoLC-MS/MS. Five upregulated and five downregulated proteins in breast cancer versus control were identified. These potential biomarkers may provide a way to predict breast cancer aggressiveness [18].

Shepard et al. used 2D SDS PAGE to study the magnitude of liver lysine acetylation in rat pairs fed ethanol and control liquid diets. These authors detected dramatic differences in the patterns between normal and ethanol fed animals by using an acetyl-lysine antibody to western blot the 2D gels. Following up with MS, a total of 40 non-nuclear proteins were identified, half from the cytosolic fraction and half from non-nuclear membranes. Interestingly, almost all the latter were from mitochondria. They hypothesized that chronic alcohol consumption leads to hyperacetylation of protective antioxidant enzymes, enhancing oxidative stress. If so, acetyltransferase inhibitors might be useful in treating alcoholic liver disease [19].

Wang et al. investigated the role of alpha synuclein ( $\alpha$-syn) in neurodegeneration. They found that $\alpha$-syn is an efficient substrate for arginyltransferase and is arginylated in vivo. Lack of arginylation leads to $\alpha$-syn aggregation with pathological consequences. This group used 2D SDS PAGE of a radiolabeled substrate to convincingly show that $\alpha$-syn is arginylated. MS was used to identify which $\alpha$-syn amino acid residues were arginylated in native mouse brain [20]. 
A
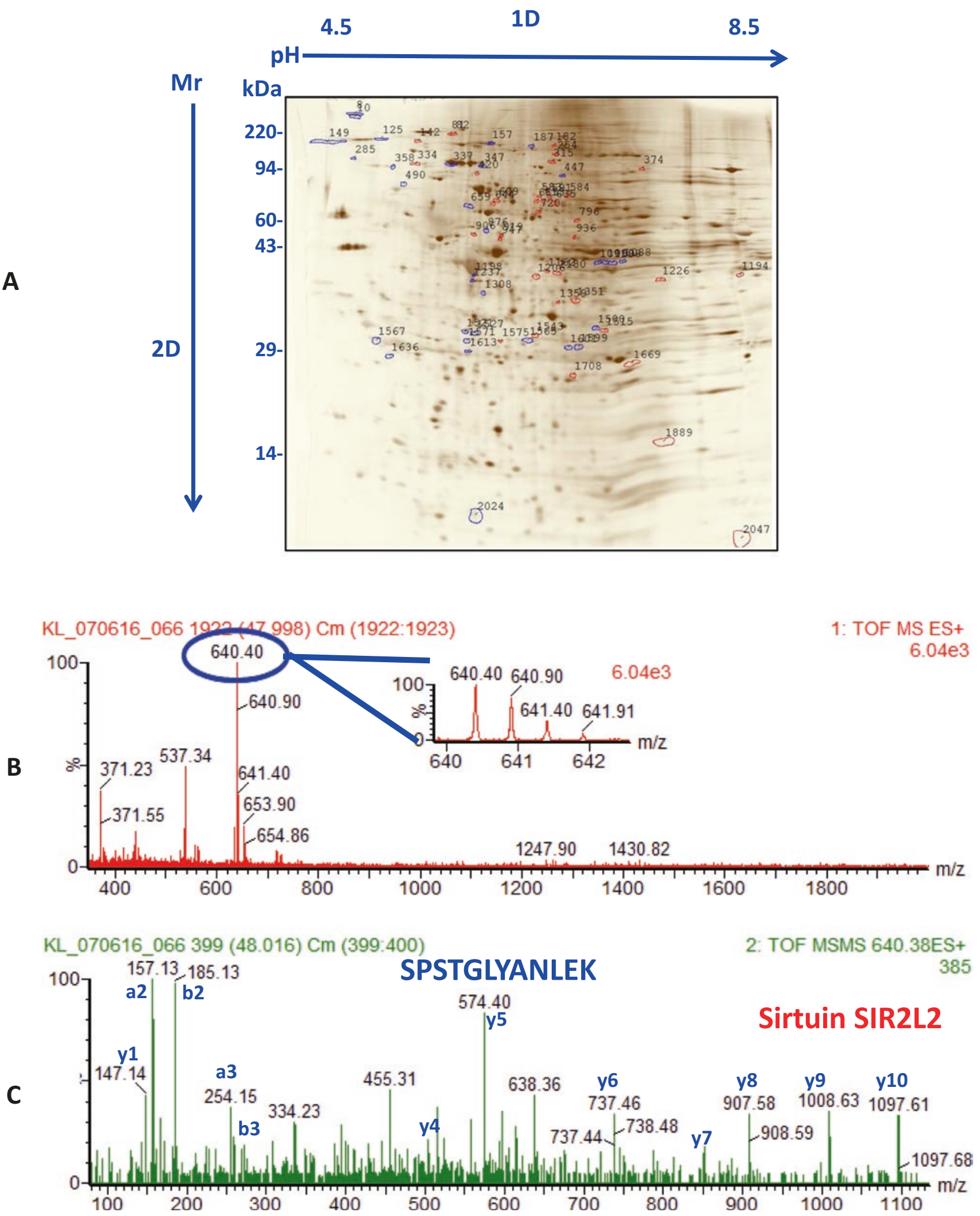

Fig. 33.6 (a) The 2D silver-stained difference pattern from an MPS 1 brain sample. Spots increased in MPS 1 versus control are outlined in blue; those increased in the control are outlined in red [13]. Spot 1351, MW $78 \mathrm{kDa}$, which increased by 4.1 -fold in the MPS 1 samples was analyzed by LC-MS/MS. (b) The MS spectrum that contains the precur- sor ion with $\mathrm{m} / \mathrm{z} 640.38(2+)$ corresponds to peptide SPSTGLYANLEK, which is part of protein Sirtuin SIR2L2. (c) The MS/MS fragmentation of precursor ion with $\mathrm{m} / \mathrm{z} 640.38(2+)$ produced a series of $\mathrm{b}$, a, and $\mathrm{y}$ ions whose analysis confirmed the sequence of peptide SPSTGLYANLEK and therefore the identity of protein Sirtuin SIR2L2 
KL_052718_022 1782(34.390) Cm (1779:1787)

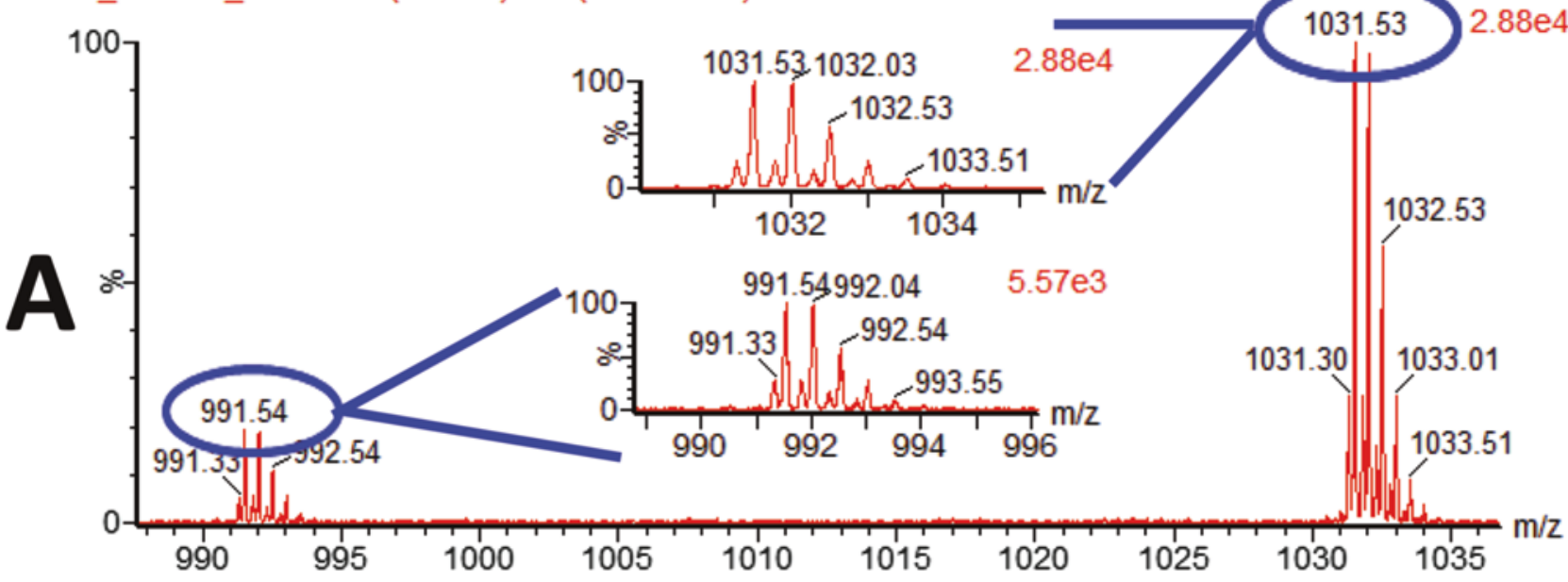

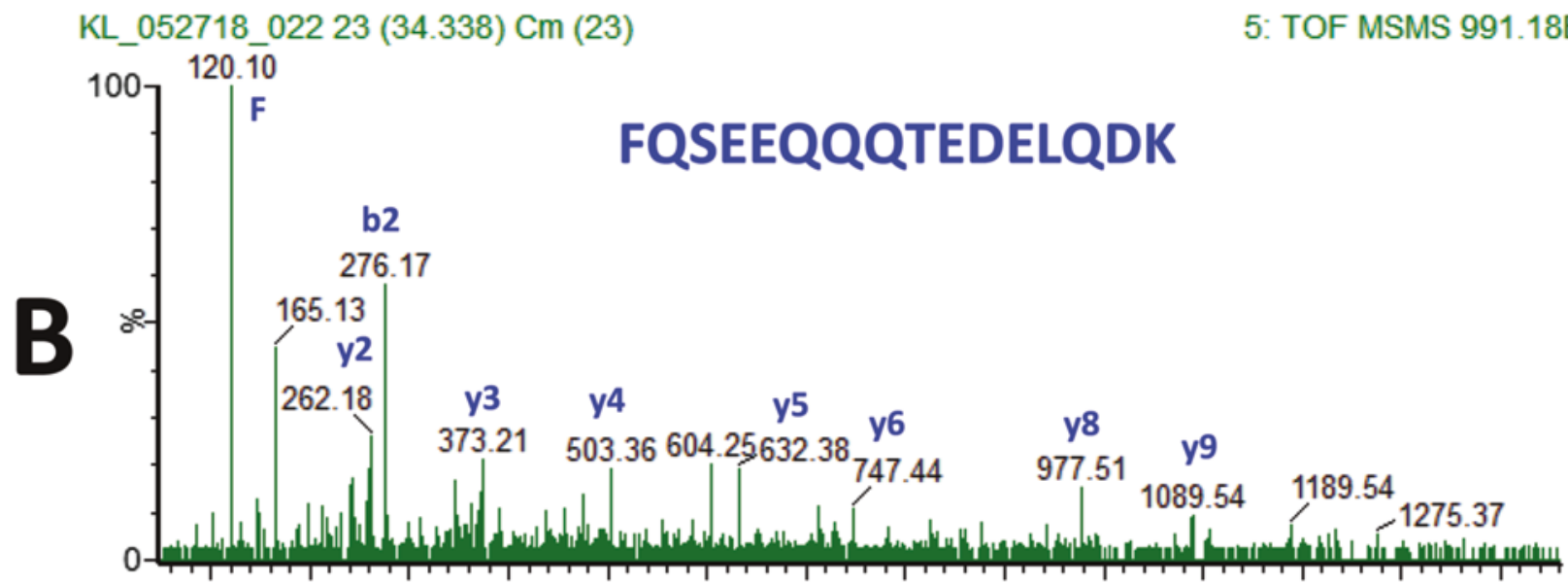

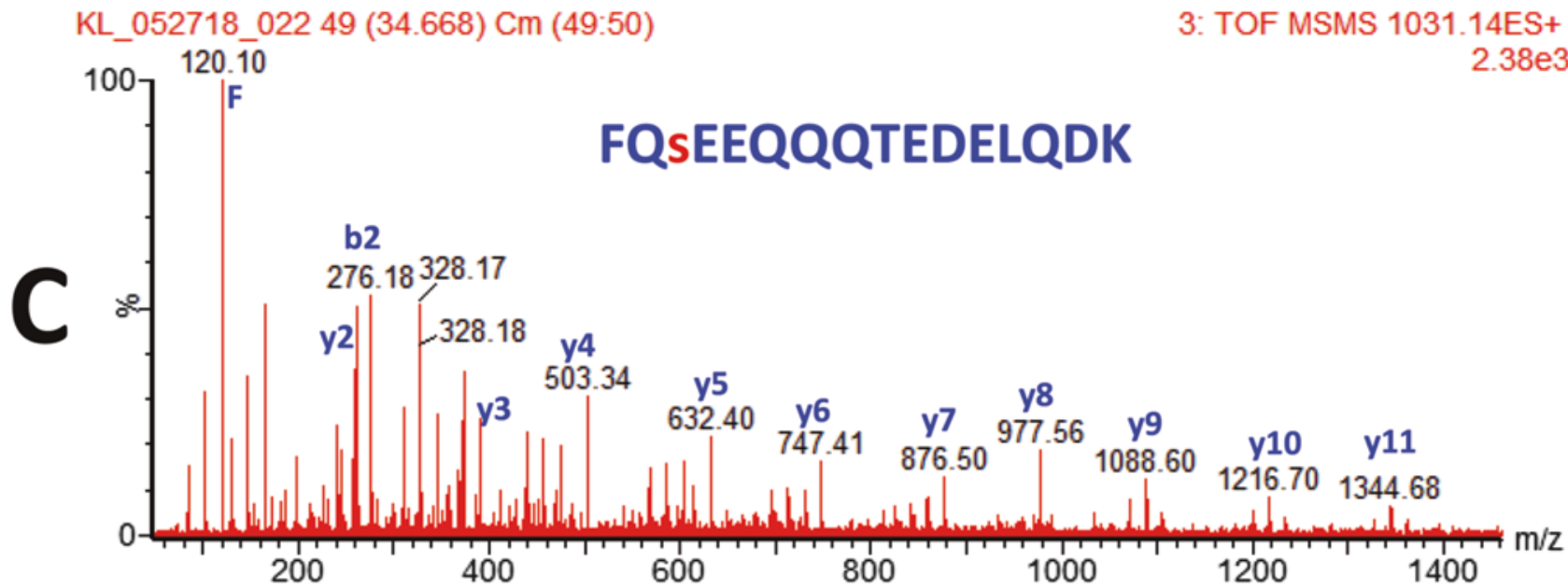

Fig. 33.7 Identification of pSer residues by MS. (a) MS spectrum showing two peaks with $\mathrm{m} / \mathrm{z} 991.54(2+)$ and 1031.53(2+), which correspond to peptide FQSEEQQQTEDELQDK (unmodified) and the same peptide phosphorylated at either serine or threonine. (b) MS/MS spectrum of the precursor ion with $\mathrm{m} / \mathrm{z} 991.54(2+)$ produced a series of $\mathrm{b}$ and $\mathrm{y}$ ions that led to identification of unmodified peptide FQSEEQQQTEDELQDK. (c) MS/MS spectrum of the precursor ion with $\mathrm{m} / \mathrm{z} 1031.53(2+)$ produced a series of $\mathrm{b}$ and $\mathrm{y}$ ions that led to identification of peptide FQSEEQQQTEDELQDK, phosphorylated at either Ser or Thr. Identification of y8, y9, y10 and y11 show clearly that the Thr residues are not phosphorylated, leaving pSer the only possibility. Therefore, not only can the amino acid sequence of a peptide be identified, but also PTMs can be identified and the modified amino acid determined. 
A

\section{AN_052215_18 $1468(33.609) \mathrm{Cm}(1463: 1468)$}

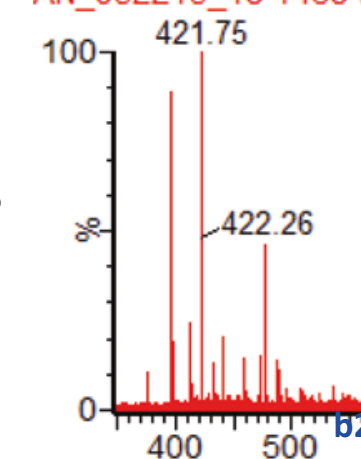

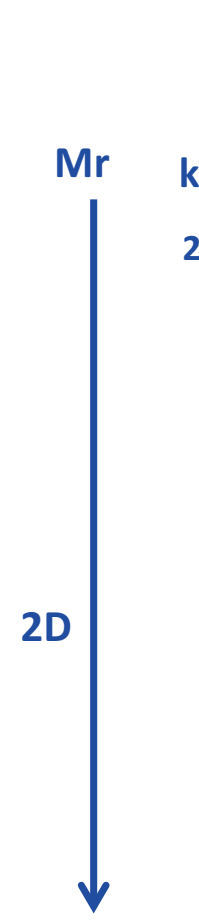

10

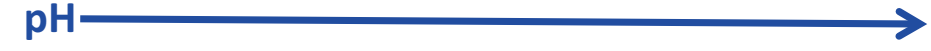

94

$60-$

43-

29-

14-

2760

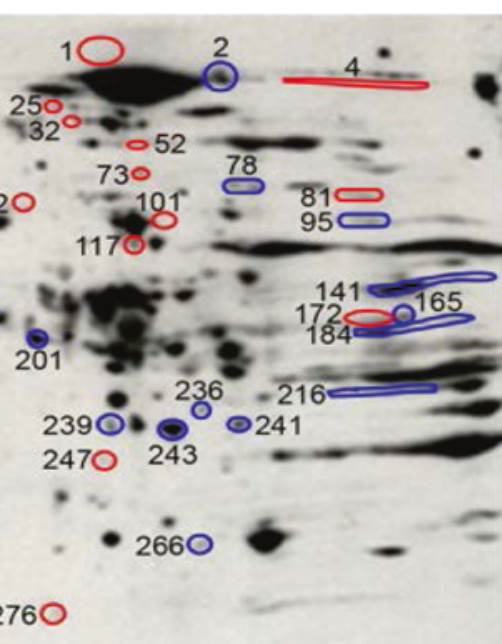

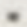

1: TOF MS ES+ 353

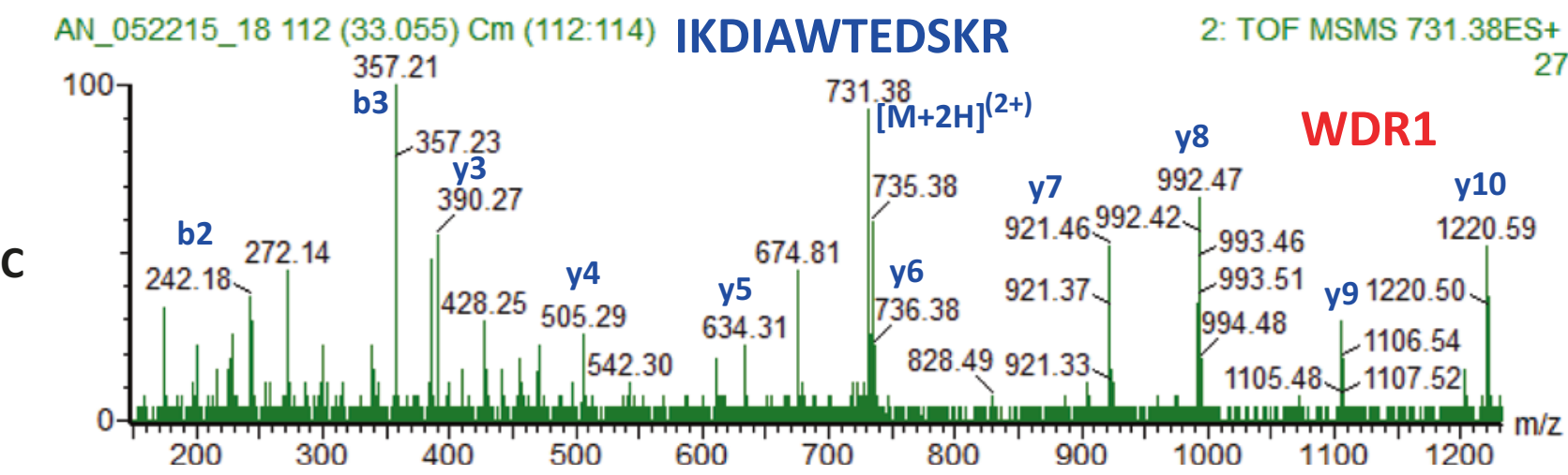

Fig. 33.8 (a) 2D SDS PAGE pSer/pThr WB of E20A (kinase-dead) cells showing spots with decreased reactivity (red outlines) relative to WT STK16 cells. Blue outlines show spots with increased pSer/pThr signal in E20A [17]. One of the former group, spot \#101 was analyzed by LC-MS/MS. (b) The MS spectrum that contains the precursor ion with $\mathrm{m} / \mathrm{z} 731.38(2+)$ corresponds to peptide IKDIAWTEDSKR, which is part of protein WDR1. (c) The MS/MS fragmentation of precursor ion with $\mathrm{m} / \mathrm{z}$ 731.38(2+) produced a series of $\mathrm{b}$ and $\mathrm{y}$ ions whose analysis confirmed the sequence of peptide IKDIAWTEDSKR and therefore the identity of protein WDR1. Note that protein WDR1 has two STK16 consensus sequences 


\subsection{Discussion and Outlook}

One way to investigate the usefulness of a method is to check citations by the scientific community over time. To that end, we searched PubMed for publications using the keyword "2D-PAGE” from 1980 to date. Results are shown in Fig. 33.9.

After O'Farrell first described the 2D method in 1975, many groups tinkered with it, trying to make it user-friendly. Flat-bed laser scanners were developed with high resolution and good linearity. Software developers searching for algorithms that automatically outlined/quantified 2D spots much preferred 2D patterns with round, tight spot outlines as occurs with cytosolic and serum proteins. The first commercial production of IPG strips, where immobilized $\mathrm{pH}$ gradients were affixed to solid supports, was a major technical breakthrough in 1991 [2]. Running 2D gels in small labs became technically much easier. The rise in papers published per year from 2000 to 2010 is almost certainly due to increased use of IPG strip 2D PAGE. In the next decade, as research focused on genomics and advances in mass spectrometry, use of IPG 2D PAGE declined.

Kendrick Labs started out in 1987 as a core lab specializing in running 2D gels for academic clients. From 1998 to 2007, we averaged 15 acknowledgements/year from PubMed papers published by academic clients with a maximum of 26 in 2006 and over 330 by 2018. As pharmaceutical companies increasingly requested 2D SDS PAGE through the years to test biologics, compliance with GLP/GMP requirements including method validation and documentation became paramount. Our team became devoted to method standardization; SOPs were written for every step. Over the past 10 years, Kendrick Labs has grown and become a contract research organization (CRO) whose main clients are pharmaceutical and biotechnology companies. Because of the ease of preparing a variety of protein samples in SDS for 2D SDS PAGE, and because IPG strips are incompatible with this detergent, we have never used the latter method.

Overall, the strength of standardized 2D SDS PAGE deserves more recognition as an orthogonal method to MS, immunohistochemistry, histology, and genomic analysis for understanding disease processes. In our opinion, no single method will suffice for the latter goal. Rather, combined results from several proverbial blind men will lead to a true description of the elephant.

Acknowledgements We thank team members of Kendrick Labs and the Biochemistry \& Proteomics Group for their hard work and fruitful discussions. We also thank Drs. Ou and Tuma for allowing use of their published results in our Figs. 33.6 and 33.8.

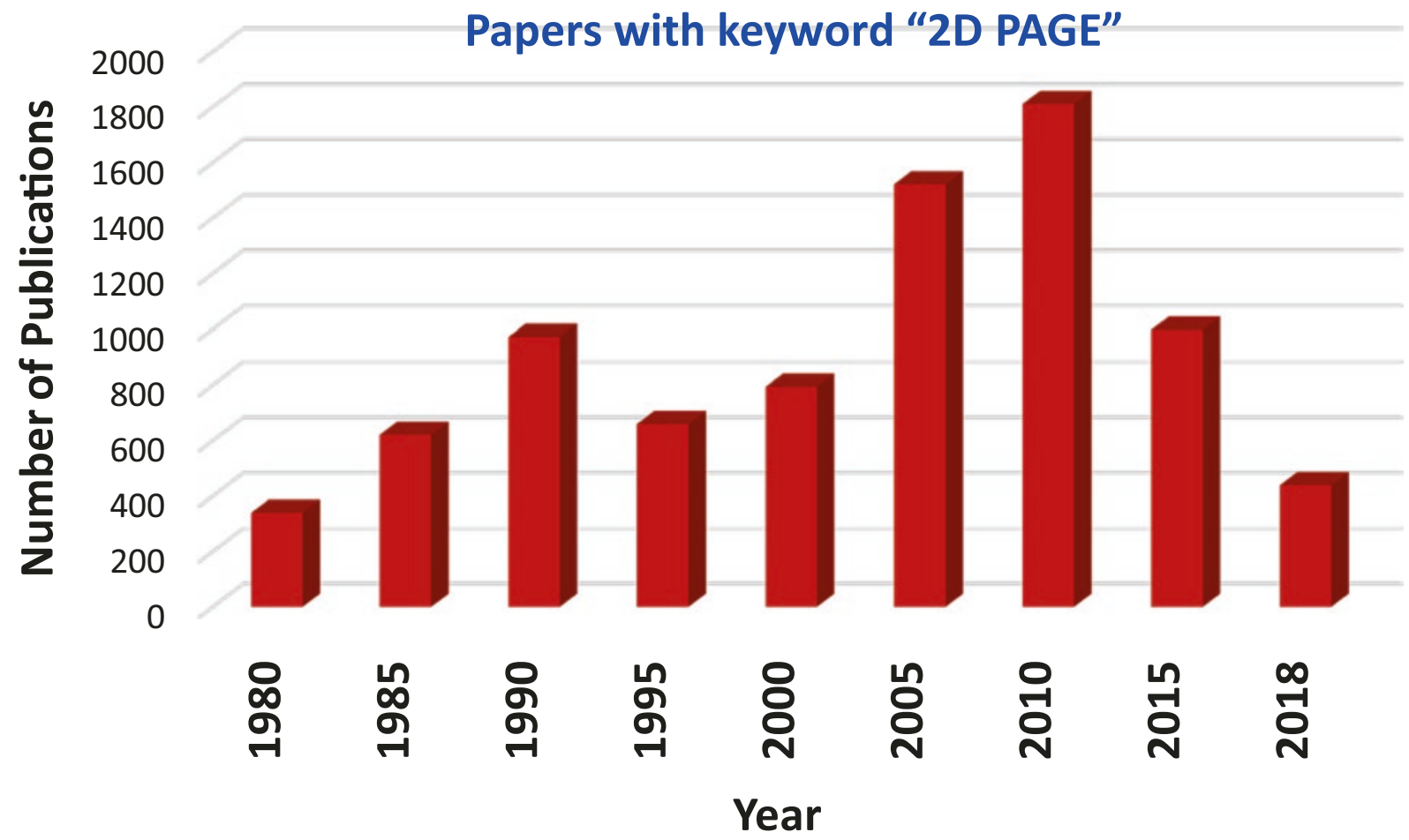

Fig. 33.9 PubMed papers/year containing the keyword "2D PAGE" checked at 5 year intervals 


\section{References}

1. O'Farrell, P. H. (1975). High resolution two-dimensional electrophoresis of proteins. The Journal of Biological Chemistry, 250, 4007-4021.

2. Gorg, A., Drews, O., Luck, C., Weiland, F., \& Weiss, W. (2009). 2-DE with IPGs. Electrophoresis, 30(Suppl. 1), S122-S132.

3. Rabilloud, T. (2009). Membrane proteins and proteomics: Love is possible, but so difficult. Electrophoresis, 30(Suppl. 1), S174-S180.

4. Dunn, M. J. (1986). Gel electrophoresis of proteins. Bristol: Wright. xiii, 407p.

5. Almen, M. S., Nordstrom, K. J., Fredriksson, R., \& Schioth, H. B. (2009). Mapping the human membrane proteome: A majority of the human membrane proteins can be classified according to function and evolutionary origin. BMC Biology, 7, 50.

6. Rabilloud, T., \& Lelong, C. (2011). Two-dimensional gel electrophoresis in proteomics: A tutorial. Journal of Proteomics, 74, 1829-1841.

7. Zhou, S., Bailey, M. J., Dunn, M. J., Preedy, V. R., \& Emery, P. W. (2005). A quantitative investigation into the losses of proteins at different stages of a two-dimensional gel electrophoresis procedure. Proteomics, 5, 2739-2747.

8. Anderson, L., \& Anderson, N. G. (1977). High resolution two-dimensional electrophoresis of human plasma proteins. Proceedings of the National Academy of Sciences of the United States of America, 74, 5421-5425.

9. Burgess-Cassler, A., Johansen, J. J., Santek, D. A., Ide, J. R., \& Kendrick, N. C. (1989). Computerized quantitative analysis of Coomassie-blue-stained serum proteins separated by twodimensional electrophoresis. Clinical Chemistry, 35, 2297-2304.

10. Anderson, N. L., \& Hickman, B. J. (1979). Analytical techniques for cell fractions. XXIV. Isoelectric point standards for twodimensional electrophoresis. Analytical Biochemistry, 93, 312-320.

11. Rodriguez, H., \& Pennington, S. R. (2018). Revolutionizing precision oncology through collaborative proteogenomics and data sharing. Cell, 173, 535-539.
12. Lux, J. C., Channaveerappa, D., Aslebagh, R., Heintz, T. A., McLerie, M., Panama, B. K., et al. (2018). Identification of dysregulation of atrial proteins in rats with chronic obstructive apnea using two-dimensional polyacrylamide gel electrophoresis and mass spectrometry. Journal of Cellular and Molecular Medicine. https://doi.org/10.1111/jcmm.14131

13. Ou, L., Przybilla, M. J., \& Whitley, C. B. (2016). Proteomic analysis of mucopolysaccharidosis I mouse brain with two-dimensional polyacrylamide gel electrophoresis. Molecular Genetics and Metabolism, 120(1-2), 101-110.

14. Walsh, C. (2006). Posttranslational modification of proteins: Expanding nature's inventory. Englewood, CO: Roberts and Co. Publishers, xxi, 490p.

15. Olsen, J. V., Vermeulen, M., Santamaria, A., Kumar, C., Miller, M. L., et al. (2010). Quantitative phosphoproteomics reveals widespread full phosphorylation site occupancy during mitosis. Science Signaling, 3, ra3.

16. Milanesi, L., Petrillo, M., Sepe, L., Boccia, A., D’Agostino, N., et al. (2005). Systematic analysis of human kinase genes: A large number of genes and alternative splicing events result in functional and structural diversity. BMC Bioinformatics, 6(Suppl. 4), S20.

17. Lopez-Coral, A., Striz, A. C., \& Tuma, P. L. (2018). A serine/threonine kinase 16-based phospho-proteomics screen identifies WD repeat protein-1 as a regulator of constitutive secretion. Scientific Reports, 8, 13049.

18. Aslebagh, R., Channaveerappa, D., Arcaro, K. F., \& Darie, C. C. (2018). Comparative two-dimensional polyacrylamide gel electrophoresis (2D-PAGE) of human milk to identify dysregulated proteins in breast cancer. Electrophoresis. https://doi.org/10.1002/ elps. 201800025

19. Shepard, B. D., Tuma, D. J., \& Tuma, P. L. (2010). Chronic ethanol consumption induces global hepatic protein hyperacetylation. Alcoholism, Clinical and Experimental Research, 34, 280-291.

20. Wang, J., Han, X., Leu, N. A., Sterling, S., Kurosaka, S., et al. (2017). Protein arginylation targets alpha synuclein, facilitates normal brain health, and prevents neurodegeneration. Scientific Reports, 7, 11323.

Open Access This chapter is licensed under the terms of the Creative Commons Attribution 4.0 International License (http://creativecommons. org/licenses/by/4.0/), which permits use, sharing, adaptation, distribution and reproduction in any medium or format, as long as you give appropriate credit to the original author(s) and the source, provide a link to the Creative Commons license and indicate if changes were made.

The images or other third party material in this chapter are included in the chapter's Creative Commons license, unless indicated otherwise in a credit line to the material. If material is not included in the chapter's Creative Commons license and your intended use is not permitted by statutory regulation or exceeds the permitted use, you will need to obtain permission directly from the copyright holder. 